1 Hacettepe Journal of Mathematics and Statistics

$\bigcap$ Volume 48 (1) (2019), 312-331

\title{
Two-stage network DEA with convex hull in intermediate products
}

\author{
Samira Maleki*, Ali Ebrahimnejad ${ }^{\dagger \ddagger}$ and Reza Kazemi Matin ${ }^{\S}$
}

\begin{abstract}
In recent Data envelopment analysis (DEA) literature, many researchers have examined systems with a two-stage network structure and its pitfalls. In these studies, two-stage network systems operations for converting inputs into outputs have been performed in two stages, meanwhile the intermediate products were considered as the outputs from the first stage and as inputs to the second stage. This duality in dealing with intermediate products imposes restrictions on the pricing of these products. In this paper by focusing on the convexity axiom, we define a new production possibility set. The main contributions of this paper are fourfold: (1) we propose models for evaluating the overall efficiency measure of decision making units (DMUs) in a two-stage network structure based on the convex hull in intermediate products; (2) we propose a procedure to determine the target unit of each inefficient DMU; (3) we explain how to calculate the divisional efficiency; (4) we demonstrate the feasibility and richness of the obtained solutions in the context of two examples.
\end{abstract}

Keywords: Data envelopment analysis (DEA), Two-stage network, Intermediate products, Convex hull, Overall efficiency, Projection.

2000 AMS Classification: $90 \mathrm{Cxx}, 90 \mathrm{C} 05$.

Received : 26.05.2017 Accepted : 28.06.2018 Doi : 10.15672/HJMS.2018.610

*Department of Mathematics, Karaj Branch, Islamic Azad University, Karaj, Iran, Email: samira.maleky@gmail.com

$\dagger^{\dagger}$ Department of Mathematics, Qaemshahr Branch, Islamic Azad University, Qaemshahr, Iran, Emails: aemarzoun@gmail.com and a.ebrahimnejad@qaemiau.ac.ir

$\ddagger$ Corresponding Author.

$\S$ Department of Mathematics, Karaj Branch, Islamic Azad University, Karaj, Iran, Email: rkmatin@kiau.ac.ir 


\section{Introduction}

Data envelopment analysis (DEA) is one of the popular and growing methods in evaluating the relative efficiency of a set of similar decision making units (DMUs). In practice, DEA performs in the presence of multi-inputs variables and multi-outputs to evaluate efficiency using a model originally proposed by Charnes et al. [1]. Later, extended DEA models were applied in different contexts (see Cook and Seiford[7]). Initial DEA methods measured the efficiency of systems without any attention to the internal structure of system operations. DEA has many applications for interpreting the productivity of complex economical and engineering systems (Ebrahimnejad et al. [8, 9], Ebrahimnejad and Bagerzadeh [10], Mottaghi et al. [15], Hatami-Marbini et al. [11], Tavana et al. [19])

Over time, researchers had more attention to system operation analysis in order to find the causes of system inefficiency. The first study with a two-stage network structure using DEA was reported by Charnes et al. [2], examining matters related to employment in the army. This two-stage network model was then used by many researchers, such as Lovell et al. [14], Seiford and Zhu [17] and Sexton and Lewis [18].

In recent years, several models have been proposed to improve efficiency measurement in two-stage network systems. Wang et al. [21] introduced a two-stage method with variable returns to scale (VRS), which in each stage considered variables independently and provided intermediate products. Rho and An [16] considered slack variables in a model that provided assessment of DMUs with weak efficiency. Kao and Hwang [12] examined the possibility of decomposition in the system's overall efficiency by considering intermediate products' weights. Tone and Tsutsui [20], using a production possibility set (PPS), introduced models based on the slack variable, and Chen et al. [5] provided a new method for determining efficient projections for inefficient DMUs. Although the main contributions of these models were improvement in measuring efficiency in a two-stage network structure, but they have many problems. For example, Chen et al. [6] reported some of the limitations in efficiency measurement related to the different behaviors occurring in the stages due to using intermediate products. Furthermore, Chen et al. [6] examined the determination of projection, efficient frontiers, and divisional efficiency become challenges in network DEA models. In all these methods, intermediate products were considered in the first stage as outputs and in the second stage as inputs with free disposability. Therefore, considering intermediate products with two different roles is caused problems within the system. In this study, we are going to have a uniform behavior with the intermediate products, when they are applied as inputs (consumer) of the second stage and outputs (products) of the first stage. For this purpose, we introduce a set of separated properties for every stage of the two-stage network, including consideration of the convex hull of the intermediate products. Models based upon this new production possibility set are presented to calculate the overall efficiency and projections. Finally, the new network DEA model is compared with similar methods in evaluating how well they addressed two-stage network limitations.

The rest of the paper is organized into several sections. In Section (2), a brief review of some systems with a two-stage network structure is presented. In Section (3), some properties regarding two-stage network DEA with convex intermediate products are explored, and $T_{C H I}$ is established by accepting these principals. Section (4) presents a new model to compute the overall efficiency score in the proposed PPS. Section (5) explored models determined by the divisional efficiency in $T_{C H I}$. In Section (6), we propose a method is proposed to improve inefficient DMUs and to calculate frontier projections in $T_{C H I}$. Some examples are illustrated in Section (7). Finally, Section (8), including the main conclusions as well as some interesting future research lines, ends the paper. 


\section{Two-stage network}

Consider Figure 1 that represents a two-stage network structure for each of a set of $n$ DMUs.

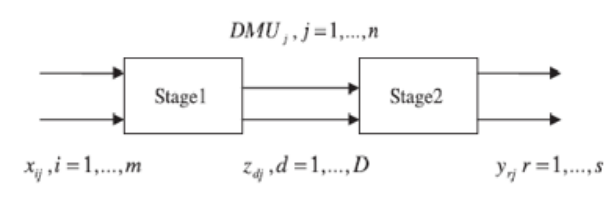

Figure 1. Two-stage process.

We apply Kao and Hwang's model [12] to explain the main concepts. For each $D M U_{j}(j=$ $1,2, \cdots, n)$, in the first stage, inputs $x_{i j},(i=1,2, \cdots, m)$ is used to produce a set of $D$, intermediate products, $z_{d j},(d=1,2, \cdots, D)$, and in the second stage all outputs of the first stage, namely, $z_{d j},(d=1,2, \cdots, D)$ is used to produce the final outputs $y_{r j},(r=1,2, \cdots, s)$. In what follows $x=\left(x_{1}, \cdots, x_{m}\right) \in R_{+}^{m}, z=\left(z_{1}, \cdots, z_{d}\right) \in$ $R_{+}^{D}, y=\left(y_{1}, \cdots, y_{s}\right) \in \mathbf{R}_{+}^{\mathbf{s}}$, represent the input vector, intermediate products vector, and output vector, respectively.

In conventional models of DEA, two different methods are commonly used to evaluate the efficiency of two-stage systems. The first method calculated efficiency of each division based upon the application of the definition of relative efficiency in a set of DMUs, and the multiplier-based network DEA models are derived according to this method. In the second method, the production possibility set is used for measuring efficiency of each division, and the envelopment models are derived with a two-stage network structure. Network DEA pitfalls were represented by applying different concepts of efficiency in these two different methods. A brief review of these two methods appears in the following subsections.

2.1. The multiplier models with two-stage network structure. The multiplierbased network models are generally applied to calculate overall and divisional efficiency. Of course, one of the limitations of the network DEA models is that the divisional efficiency envelopment models are infeasible in some cases. Kao and Hwang [12], under constant returns to scale (CRS) assumption, calculated stages' efficiency scores separately, then considering a series relationship between stages, they obtained an overall efficiency score by the products of each stages' efficiency. One notable point in their method is that the weights related to intermediate products are equal in both stages $\left(w_{d} ; d=1, \cdots, D\right)$.

Kao and Hwang [12] proposed the following linear programming (LP) model to evaluate the overall efficiency measure for $D M U_{o}$ :

$$
\begin{aligned}
E_{o}=\max & \sum_{r=1}^{s} u_{r} y_{r o} \\
\text { s.t. } \quad & \sum_{r=1}^{s} u_{r} y_{r j}-\sum_{d=1}^{D} w_{d} z_{d j} \leq 0 \quad j=1, \cdots, n \\
& \sum_{d=1}^{D} w_{d} z_{d j}-\sum_{i=1}^{m} v_{i} x_{i j} \leq 0 \quad j=1, \cdots, n
\end{aligned}
$$




$$
\begin{aligned}
& \sum_{i=1}^{m} v_{i} x_{i o}=1 \\
& v_{i} \geq 0, u_{r} \geq 0, w_{d} \geq 0, i=1, \cdots, m ; r=1, \cdots, s ; d=1, \cdots, D
\end{aligned}
$$

In model (2.1), $u \in R_{+}^{s}, v \in R_{+}^{m}$ and $w \in R_{+}^{D}$ are the associated unknown weights of the output, input and intermediate products, respectively. Kao and Hwang [13] also provided a method for calculating the overall efficiency score of $D M U_{o}$ under VRS. They introduced following models for calculating the divisional efficiency scores.

$$
\begin{aligned}
& T_{o}^{1}=\max \sum_{d=1}^{D} w_{d} z_{d o}-\dot{w}_{0} \\
& \text { s.t. } \quad \sum_{i=1}^{m} v_{i} x_{i o}=1 \\
& E_{o} \sum_{i=1}^{m} v_{i} x_{i o}-\sum_{r=1}^{s} u_{r} y_{r o}=0 \\
& \sum_{r=1}^{s} u_{r} y_{r j}-\sum_{d=1}^{D} w_{d} z_{d j} \leq 0 \quad j=1, \cdots, n \\
& \sum_{d=1}^{D} w_{d} z_{d j}-\sum_{i=1}^{m} v_{i} x_{i j} \leq 0 \quad j=1, \cdots, n \\
& \sum_{d=1}^{D} \dot{w}_{d} z_{d j}-\dot{w}_{0}-\sum_{i=1}^{m} v_{i} x_{i j} \leq 0 \quad j=1, \cdots, n \\
& u_{r}, v_{i}, w_{p}, w_{p} \geq \varepsilon \\
& w_{0} \text { free in sign } \\
& T_{o}^{2}=\max \sum_{r=1}^{s} u_{r} y_{r o} \\
& \text { s.t. } \sum_{d=1}^{D} \dot{w}_{d} z_{d o}+\dot{w}_{0}=1 \\
& E_{o} \sum_{i=1}^{m} v_{i} x_{i o}-\sum_{r=1}^{s} u_{r} y_{r o}=0 \\
& \sum_{r=1}^{s} u_{r} y_{r j}-\sum_{d=1}^{D} w_{d} z_{d j} \leq 0 \quad j=1, \cdots, n \\
& \sum_{d=1}^{D} w_{d} z_{d j}-\sum_{i=1}^{m} v_{i} x_{i j} \leq 0 \quad j=1, \cdots, n \\
& \sum_{r=1}^{s} u_{r} y_{r j}-\sum_{d=1}^{D} \dot{w}_{d} z_{d j}-\dot{w}_{0} \leq 0 \quad j=1, \cdots, n \\
& u_{r}, v_{i}, w_{p}, \dot{w}_{p} \geq \varepsilon \\
& w_{0} \text { free in sign }
\end{aligned}
$$

Hence, $E_{o}$ is the overall efficiency score under CRS calculated from model (2.1). Model (2.2) computes the input-oriented technical efficiency score of the first step and model 
(2.3) evaluates the output-oriented efficiency score of the second stage for $D M U_{o}$. The overall efficiency score of $D M U_{o}$, under VRS, is obtained via the products of the stages' efficiency scores.

Chen et al. [3] calculated the overall efficiency in a two-stage system by use of specific weights in the objective function. They proposed the following model to compute the VRS's overall efficiency in a two-stage system, when $D M U_{o}$ is under evaluation:

$$
\begin{array}{ll}
\max & \sum_{d=1}^{D} \mathrm{w}_{d} z_{d o}+u^{1}+\sum_{r=1}^{s} u_{r} y_{r o}+u^{2} \\
\text { s.t. } & \sum_{r=1}^{s} u_{r} y_{r j}-\sum_{d=1}^{D} w_{d} z_{d j}+u^{2} \leq 0 \quad j=1, \cdots, n \\
& \sum_{d=1}^{D} w_{d} z_{d j}-\sum_{i=1}^{m} \mathrm{v}_{i} x_{i j}+u^{1} \leq 0 \quad j=1, \cdots, n \\
& \sum_{i=1}^{m} v_{i} x_{i o}+\sum_{d=1}^{D} \mathrm{w}_{d} z_{d o}=1 \\
& v_{i} \geq 0, u_{r} \geq 0, w_{d} \geq 0, i=1, \cdots, m ; r=1, \cdots, s ; d=1, \cdots, D \\
& u^{1}, u^{2} \text { free in sign }
\end{array}
$$

As can be seen form Model (2.4), intermediate products' weights are considered the same in both stages of the proposed model.

2.2. Envelopment models with two-stage network structure. DEA models with network structures are used for determining projections on the efficiency frontier. Chen et al. [4] introduced a radial version of the envelopment-based network model to compute the input-oriented CRS overall efficiency for $D M U_{o}$ as follows:

$$
\begin{array}{ll}
\min \theta & \\
\text { s.t. } & \sum_{j=1}^{n} \lambda_{j} x_{i j} \leq \theta x_{i o}, \quad i=1,2, \cdots, m \\
& \sum_{j=1}^{n} \lambda_{j} z_{d j} \geq \tilde{z}_{d o}, \quad d=1,2, \cdots, D \\
& \sum_{j=1}^{n} \mu_{j} z_{d j} \leq \tilde{z}_{d o}, \quad d=1,2, \cdots, D \\
& \sum_{j=1}^{n} \mu_{j} y_{r j} \geq y_{r o}, \quad r=1,2, \cdots, s \\
& \lambda_{j} \geq 0, \mu_{j} \geq 0 \quad j=1,2, \cdots, n \\
& \tilde{z}_{d o} \geq 0 \quad d=1,2, \cdots, D
\end{array}
$$

Model (2.5), is equivalent to the dual of Model (2.1). In Model (2.5), the intermediate products are treated as free links.

Chen et al. [5] applied redundant constraints of Model (2.5), such as $\tilde{z}_{d o} \geq 0(d=$ $1,2, \cdots, D)$ in Model $(2.5)$ as unrestricted variables, i.e. $\tilde{z}_{d o}(d=1,2, \cdots, D)$ are free in sing. Therefore, in the dual model, both constraints of intermediate products corresponding to these free variables considered as equal constraints. For reformulating Model (2.5) under the assumption of VRS, it is enough to add the convexity constraints $\left(\sum_{j=1}^{N} \mu_{j}=\sum_{j=1}^{N} \lambda_{j}=1\right)$ to model (2.5). 
Tone and Tsutsui [20] introduced slacks-based network DEA models by using production possibility set. They explored several models based upon the intermediate products as both fixed links and free links. A version of the input-oriented envelopment-based network model, where the intermediate products referred to as free link cases can be modeled as follows:

$$
\begin{array}{ll}
\max & \sum_{i=1}^{m} \frac{s_{i}^{-}}{x_{i o}} \\
\text { s.t. } & \sum_{j=1}^{n} \lambda_{j} x_{i j}+s_{i}^{-}=x_{i o}, \quad i=1, \cdots, m \\
& \sum_{j=1}^{n} \lambda_{j} z_{d j} \geq \tilde{z}_{d o}, \quad d=1, \cdots, D \\
& \sum_{j=1}^{n} \mu_{j} z_{d j} \leq \tilde{z}_{d o}, \quad d=1, \cdots, D \\
& \sum_{j=1}^{n} \mu_{j} y_{r j}-s_{r}^{+}=y_{r o}, \quad r=1, \cdots, s \\
& \lambda_{j} \geq 0, \quad \mu_{j} \geq 0, \quad j=1, \cdots, n \\
& \tilde{z}_{d o} \geq 0, \quad d=1, \cdots, D
\end{array}
$$

In general, two different behaviors with intermediate products in most models of a twostage network can be considered one of their most significant problems while minimum attention has been paid to them. These methods by assigning the same weight variables to the intermediate products in two stages of the multiplier-based network models (for example, $\left.\mathrm{w}_{d} ; d=1, \ldots, D\right)$ impose uniform shadow prices to the system and so they have limitations or have less flexibility.

By changing the direction of inequality in the corresponding constraints of intermediate products in the envelopment network models, the model provides the possibility of disposability, which is consumed in the next stage. Therefore, models manage the problem from the outside. In the next section, we examine the impact of uniform behavior on these products for calculating overall efficiency and projections.

\section{Two-stage network DEA with convex intermediate products}

In this section, we propose separate axioms for each stage in a two-stage network structure. Using these axioms, we form a new production possibility set for a twostage network DEA with convex intermediate products. In addition, some of the related properties are also presented.

We postulate the following axioms for the production possibility set of the first stage:

A1 . The observed activities $\left(x_{j}, z_{j}\right),(j=1,2, \cdots, n)$ belong to $T_{1}$.

A2 Any convex combination of activities in $T_{1}$ belongs to $T_{1}$.

A3 .For an activity $(x, z)$, in $T_{1}$, any semi positive activity $(\bar{x}, z)$ with $\bar{x} \geq x$ is included in $T_{1}$.

Thus, we define the production possibility set $T_{1}$ that satisfiesA1-A3 as follows:

$$
T_{1}=\left\{(x, z): \sum_{j} \lambda_{j} x_{j} \leq x, \sum_{j} \lambda_{j} z_{j}=z, \sum_{j} \lambda_{j}=1, \lambda_{j} \geq 0 ; j=1, \cdots, n\right\}
$$

In PPS $T_{1}$, the variable $\lambda \in R^{n}$ is the vector of intensity variables of the first stage. 
3.1. Theorem. Technologyof the first stage, $T_{1}$, which is defined in set (3.1) is the minimal set that contains all observations and satisfies the axioms of strong disposability of inputs and convexity.

The proof of Theorem (3.1) is given in the Appendix.

For the PPS of the second stage, we postulate the following axioms:

B1 . The observed activities $\left(z_{j}, y_{j}\right),(j=1,2, \cdots, n)$ belong to $T_{2}$.

B2 . Any convex combination of activities in $T_{2}$ belongs to $T_{2}$.

B3 . For an activity $(z, y)$, in $T_{2}$, any semi positive activity $(z, \bar{y})$ with $y \geq \bar{y}$ is included in $T_{2}$.

Then, we define the production possibility set $T_{2}$ that satisfies B1-B3 as follows:

$$
T_{2}=\left\{(z, y): \sum_{j} \mu_{j} z_{j}=z, \sum_{j} \mu_{j} y_{j} \geq y, \sum_{j} \mu_{j}=1, \mu_{j} \geq 0 ; j=1, \cdots, n\right\}
$$

In PPS $T_{2}$, the variables $\mu \in R^{n}$ denote the intensity levels of the DMUs for the second stage.

3.2. Theorem. The second stage technology $T_{2}$ defined in (3.2) is the minimal set that contains all observations and satisfies the axioms of strong disposability of outputs and convexity.

The proof of Theorem (3.2) is similar to the proof of Theorem (3.1) and is omitted.

According to the proposed axioms for each stage, and under the assumption of VRS, we define the overall production possibility set for the two-stage network with convex intermediate products as follows:

$$
\begin{aligned}
T_{C H I}= & \left\{(x, z, y): \sum_{j} \lambda_{j} x_{j} \leq x, \sum_{j} \lambda_{j} z_{j}=z, \sum_{j} \mu_{j} z_{j}=z,\right. \\
& \sum_{j} \mu_{j} y_{j} \geq y, \sum_{j} \mu_{j}=1, \sum_{j} \lambda_{j}=1, \lambda_{j} \geq 0, \\
& \left.\mu_{j} \geq 0, \quad j=1, \ldots, n\right\}
\end{aligned}
$$

In fact, notation $C H I$ represents the convex hull of intermediate products.

Note that the intermediate products in $T_{C H I}$ are examined by two separate sets of $\lambda \in R^{n}$ and $\mu \in R^{n}$, and thus $\lambda$ determines the relation between inputs and intermediate products and $\mu$ determines the relation between intermediate products and outputs.

The main difference of the technology expressed in (3.3) from the conventional technology of the two-stage network is that the former allows the free disposability for the intermediate products.

In the above technology, the produced output ratio in the first stage is equal to the consumed input ratio to the second stage. Therefore, access to resources became restricted, and the produced possibility set generated by technology set (3.3) becomes a subset of the traditional two-stage network production possibility set.

Here, we present an illustrative example to compare the overall efficiency frontier and each stage of the two-stage system, using efficiency frontier conventional technology under the condition of CRS and VRS.

3.3. Example. Consider a system that includes four DMUs. Each DMU has one input, one output, and one intermediate measure. The data set is given in Table 1.

Now, we can show the overall production technology, and PPS of each stage of the two-stage structure as seen in Figure 2, Figure 3, and Figure 4. 
Table 1. Data set of example (3.3)

\begin{tabular}{|c|c|c|c|}
\hline DMU & $\boldsymbol{x}$ & $\mathbf{z}$ & $\mathbf{y}$ \\
\hline $\mathbf{A}$ & 1 & 2 & 4 \\
\hline $\mathbf{B}$ & 2 & 1 & 1 \\
\hline $\mathbf{C}$ & 5 & 4 & 2 \\
\hline $\mathbf{D}$ & 2 & 2 & 2 \\
\hline
\end{tabular}

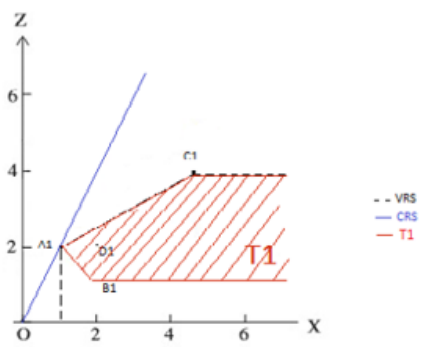

Figure 2. Efficient frontier generated by $T_{1}$.

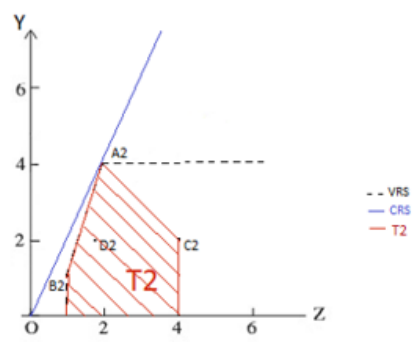

Figure 3. Efficient frontier generated by $T_{2}$.

In Figure 2, the red lines show the production frontier of stage $1\left(T_{1}\right)$. Note that the efficiency frontier has been expanded by the convex hull of observations and the strong disposability in inputs.

In Figure 3, the red lines show the production frontier of stage $2\left(T_{2}\right)$, in which the frontier points are obtained with the convex hull of observations and the strong disposability axiom in outputs. The blue lines and black dotted lines on both Figures 2 and 3 represent the efficiency frontier, under the assumptions of CRS and VRS, respectively. Clearly, the production possibility set with the convex hull in intermediate products is a subset of the production possibility set under the assumption of both CRS and VRS.

Figure 4 illustrate the tridimensional network technology showing the convex hull in intermediate products.

\section{Introducing a model to determine overall efficiency in $\mathbf{T}_{\mathbf{C H I}}$}

In this section, we present a new network DEA model to calculate the overall efficiency in $T_{C H I}$. To do this, we first consider an input-oriented model. 


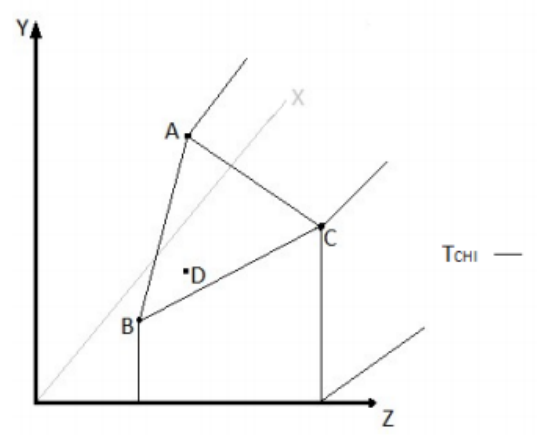

Figure 4. Efficient frontier generated by $T_{C H I}$.

The new network model suggested to evaluate the overall efficiency of $D M U_{o}$ in $T_{C H I}$ is given as follows:

$$
\begin{aligned}
& \theta_{o}=\min \theta \\
& \text { s.t. } \sum_{j=1}^{n} \lambda_{j} x_{i j} \leq \theta x_{i o} \quad i=1, \cdots, m \\
& \sum_{j=1}^{n} \lambda_{j} z_{d j}=z_{d o} \quad d=1, \cdots, D \\
& \sum_{j=1}^{n} \mu_{j} z_{d j}=z_{d o} \quad d=1, \cdots, D \\
& \sum_{j=1}^{n} \mu_{j} y_{r j} \geq y_{r o} \quad r=1, \cdots, s \\
& \sum_{j=1}^{n} \mu_{j}=\sum_{j=1}^{n} \lambda_{j}=1 \\
& \lambda_{j} \geq 0 \quad, \mu_{j} \geq 0 \quad j=1, \cdots, n
\end{aligned}
$$

Model (4.1) is similar to the VRS two-stage network model of Chen et al. [4] given in model (2.5),with the difference that in the above model the constraints related to intermediate products are considered in a convex set of intermediate data. In fact, outputs of the first stage are exactly equal to the inputs of the second stage and the overall efficiency of $D M U_{o}$ is evaluated into a set of fixed intermediate products. It can be seen that model (4.1) is always feasible, and $0<\theta_{o}^{*} \leq 1$.

4.1. Definition (Input-oriented overall efficiency). The under evaluation $D M U_{o}$ is said to be overall input-efficient with intermediate convex products, if the optimal value of model (4.1) is equal to one; namely $\theta_{o}^{*}=1$. 
In order to describe model (4.1), we can rewrite it to evaluate the overall efficiency score for $D M U_{B}$ given in example 3.3 as follows:

$$
\begin{aligned}
\theta_{o}^{B}=\min \theta & \\
\text { s.t. } & \lambda_{1}+2 \lambda_{2}+5 \lambda_{3}+2 \lambda_{4} \leq 2 \theta \\
& 2 \lambda_{1}+\lambda_{2}+4 \lambda_{3}+2 \lambda_{4}=1 \\
& \lambda_{1}+\lambda_{2}+\lambda_{3}+\lambda_{4}=1 \\
& 2 \mu_{1}+\mu_{2}+4 \mu_{3}+2 \mu_{4}=1 \\
& 4 \mu_{1}+\mu_{2}+2 \mu_{3}+2 \mu_{4} \geq 1 \\
& \mu_{1}+\mu_{2}+\mu_{3}+\mu_{4}=1 \\
& \lambda_{j} \geq 0, \mu_{j} \geq 0 \quad j=1, \ldots, 4
\end{aligned}
$$

By solving this model, the optimal overall efficiency score for $\mathrm{DMU}_{\mathrm{B}}$ is achieved as $\theta_{o}^{B *}=1$.

Similarly, the new model can evaluate the overall efficiency scores for the inputoriented units in Example 3.3. The results of this calculation are reported in Table 2.

Table 2. Overall efficiency scores for four DMUs in example 3.3

\begin{tabular}{|c|c|c|c|c|}
\hline DMU & A & B & C & D \\
\hline $\boldsymbol{\theta}_{\boldsymbol{o}}^{*}$ & 1 & 1 & 1 & 0.5 \\
\hline
\end{tabular}

Thus, DMUs A, B, and C are efficient. Note that the obtained scores for overall efficiency were in keeping with the level of intermediate products computed at the level $z_{o}$.

If we allow intermediate products in model (4.1) to change the convex hull, then further improvements in the optimal solution would be possible. We expressed this improvement in Section 6 as free intermediate products.

The dual of Model (4.1) (multiplier formation) can be presented as follows:

$$
\begin{array}{ll}
\max & \sum_{r=1}^{s} u_{r} y_{r o}+\sum_{d=1}^{D} w_{d}^{1} z_{d o}-\sum_{d=1}^{D} w_{d}^{2} z_{d o}+u_{0}+v_{0} \\
\text { s.t. } & \sum_{d=1}^{D} w^{1}{ }_{d} z_{d j}-\sum_{i=1}^{m} \mathrm{v}_{i} x_{i j}+v_{0} \leq 0 \quad j=1, \ldots, n \\
& \sum_{r=1}^{s} u_{r} y_{r j}-\sum_{d=1}^{D} w_{d}^{2} z_{d j}+u_{0} \leq 0 \quad j=1, \ldots, n \\
& \sum_{i=1}^{m} \mathrm{v}_{i} x_{i o}=1 \\
& \mathrm{v}_{i} \geq 0, \quad u_{r} \geq 0 \quad m ; r=1, \cdots, s \\
& u_{0}, v_{0}, w^{2}, w^{1} \text { free in sign }
\end{array}
$$

In Model (4.2) $w^{1}$ and $w^{2}$ denote the weights of the intermediate products in the first stage and second stage, respectively, and $v_{0}, u_{0}, u, v$ denotes unknown weights. It is trivial that the objective function value of this model is less than or equal to 1. 
Model (4.2) can be rewritten as following fractional model:

$$
\begin{aligned}
\max & \frac{\sum_{r=1}^{s} u_{r} y_{r o}+\sum_{d=1}^{D}\left(w_{d}^{1}-w_{d}^{2}\right) z_{d o}+u_{0}+v_{0}}{\sum_{i=1}^{m} \mathrm{v}_{i} x_{i o}=1} \\
\text { s.t. } & \frac{\sum_{d=1}^{D} w_{d}^{1} z_{d j}+v_{0}}{\sum_{i=1}^{m} \mathrm{v}_{i} x_{i o}=1} \leq 1 \quad j=1, \cdots, n \\
& \frac{\sum_{r=1}^{s} u_{r} y_{r j}+u_{0}}{\sum_{d=1}^{D} w^{2} z_{d j}} \leq 1 \quad j=1, \cdots, n \\
& \mathrm{v}_{i} \geq 0, u_{r} \geq 0 \quad i=1, \cdots, m ; r=1, \cdots, s \\
& u_{0}, v_{0}, w^{2}, w^{1} \quad \text { free in sign }
\end{aligned}
$$

The first and second sets of constraints in Model (4.3) show the relative efficiency of input-oriented units for $D M U_{o}$ in the first stage and the second stage, respectively. It should be noted that in the objective function of Model (4.3), the value $\left(w^{1}-w^{2}\right)$ in the numerator gives two different roles for $z_{o}$. If this value is non-negative, the performance of $z_{o}$ as output is more effective; otherwise, it is used as input.

By considering equal sign in the constraints related to intermediate products of Model (4.1), the dual variables corresponding to these constraints, namely $w^{1}$ and $w^{2}$ have no restriction signs. This means that the intermediate values can be measured with positive, negative, or even zero amounts. Thus, the system is allowed to measure intermediate products with different patterns, without considering their input or output roles. Therefore, the new model does not restrict the pricing of intermediate products.

However, in model (2.1), proposed by Kao and Hwang [12], which is equivalent to the dual of model (2.5) ([4]), same non-negative weights are assigned to two intermediate products constraints. Therefore, the obtained values of the intermediate products in model (2.1) had less flexible and imposed targeted pricing methods on the system. In fact, model (4.2) is somewhat similar to model (2.4) ([3]), with the difference that in model (2.4) the same positive weights are assigned to the intermediate products and considering predetermined weights by the decision-maker in model (2.4) are caused differences in the constraints of the normalized equations of these two models.

It should be noted that model (4.1) can also be used to assess the overall efficiency in output-oriented units. The difference is that we should replace the minimum contraction in inputs with the maximum expansion in outputs.

\section{Divisional metricconverterProductID0000019Fefficiency in $\mathbf{T}_{\mathbf{C H I}}$}

For calculating the divisional efficiency, we use the production possibility sets (3.1) and (3.2). The efficiency scores in input-oriented units for $D M U_{o}$ in the first stage, and its dual model can be computed by the following models:

$$
\begin{aligned}
& \theta_{1}=\min \theta \\
& \text { s.t. } \quad \sum_{j=1}^{n} \lambda_{j} x_{i j} \leq \theta x_{i o} \\
& i=1, \cdots, m \\
& \sum_{j=1}^{n} \lambda_{j} z_{d j}=z_{d o} \\
& d=1, \cdots, D \\
& \sum_{j=1}^{n} \lambda_{j}=1 \quad \lambda_{j} \geq 0 \\
& j=1, \cdots, n
\end{aligned}
$$




$$
\begin{aligned}
& D_{1}=\max \sum_{d=1}^{D} w_{d}^{1} z_{d o}+v_{0} \\
& \text { s.t. } \sum_{d=1}^{D} w_{d}^{1} z_{d j}-\sum_{i=1}^{m} \mathrm{v}_{i} x_{i j}+v_{0} \leq 0 \quad j=1, \ldots, n \\
& \sum_{i=1}^{m} \mathrm{v}_{i} x_{i o}=1 \\
& \mathrm{v}_{i} \geq 0 \quad i=1, \ldots, m \\
& v_{0}, w^{1} \text { free in sign }
\end{aligned}
$$

In addition, the efficiency scores for input-oriented units in the second stage can be obtained by the following models which are dual of each other:

$$
\begin{aligned}
& \theta_{2}=\min \theta \\
& \text { s.t. } \sum_{j=1}^{n} \mu_{j} z_{d j}=\theta z_{d o} \quad d=1, \cdots, D \\
& \sum_{j=1}^{n} \mu_{j} y_{r j} \geq y_{\text {ro }} \quad r=1, \cdots, s \\
& \sum_{j=1}^{n} \mu_{j}=1 \\
& \mu_{j} \geq 0 \quad j=1, \cdots, n \\
& D_{2}=\max \sum_{r=1}^{s} u_{r} y_{r o}+u_{0} \\
& \text { s.t. } \sum_{r=1}^{s} u_{r} y_{r j}-\sum_{d=1}^{D} w_{d}^{2} z_{d j}+u_{0} \leq 0 \quad j=1, \ldots, n \\
& \sum_{d=1}^{D} w_{d}^{2} z_{d o}=1 \\
& u_{r} \geq 0 \\
& r=1, \ldots, s \\
& u_{0}, w^{2} \\
& \text { free in sign }
\end{aligned}
$$

The interpretation of the models (5.3) and (5.4) are similar to standard models of DEA. The only difference is using the equality restriction in the output constraints of the first step of model (5.1) and in the input constraints of model (5.3). The dual variables corresponding to these constraints are free in sign, but the objective function values of models (5.1) and (5.3) are between zero and one, so based on the duality theorem, the problem is always bounded.

In general, input-based (output-based) models with a two-stage network structure given based on the concept of convex hull in intermediate products do not give information about divisional efficiency. This challenge may be due to using fixed intermediate products, or not using the optimal intermediate products in computation of the divisional efficiency. Indeed, the efficiency score of the first stage may represent the overall efficiency score. 


\section{Frontier projection in $\mathbf{T}_{\mathbf{C H I}}$}

In this section we introduce a new network DEA model that gives the efficient projection of inefficient DMUs.

Note that according to the multiplier-based network DEA models, it is not possible to determine the efficiency frontier or the frontier projection of units under assessment. Thus, it is not possible to determine the amount of saving in inputs while keeping the current outputs and also to determine the amount of maximum increased outputs with fixed input values.

Chen et al. [5] expressed that the resulting projections of the dual model (2.1) fail on the efficiency frontier. Therefore, they proposed model (2.5), which is equivalent to the dual of model (2.1). The key point of their model was to modify data of frontier projections with proper adjustments to the intermediate products. They replaced constraints related to intermediate measures in dual of model (2.1) with two sets of constraints, so that the right side of both constraints were replaced with a set of non-negative variables $\left(\tilde{z}_{d o}\right)$. This model not also provides the frontier projections for inefficient DMUs, but also gives an overall efficiency score.

In order to determine the frontier projections in $T_{C H I}$, we replace the amounts on the right side in both intermediate products constraints in model (4.1) with a set of the same non-negative variables. In this case we propose the following linear programming model:

$$
\begin{array}{ll}
\min \theta & \\
\text { s.t. } & \sum_{j=1}^{n} \lambda_{j} x_{i j} \leq \theta x_{i o} \quad i=1, \cdots, m \\
& \sum_{j=1}^{n} \lambda_{j} z_{d j}=\tilde{z}_{d o} \quad d=1, \cdots, D \\
& \sum_{j=1}^{n} \mu_{j} z_{d j}=\tilde{z}_{d o} \quad d=1, \cdots, D \\
& \sum_{j=1}^{n} \mu_{j} y_{r j} \geq y_{r o} \quad r=1, \cdots, s \\
& \sum_{j=1}^{n} \mu_{j}=\sum_{j=1}^{n} \lambda_{j}=1 \\
& \lambda_{j}, \mu_{j} \geq 0 \quad j=1, \cdots, n \\
& \tilde{z}_{d o} \geq 0 \quad d=1, \cdots, D
\end{array}
$$

Note that in the Model (6.1), $\tilde{z}_{\text {do }}$ denotes an unknown variable. It indicates an optimal amount of intermediate products, produced in the first stage and consumed in the second stage. The projection point for $D M U_{o}$ is given based upon optimal solution ofModel $(6.1)$ as $\left(\theta^{*} x_{i o} \tilde{z}_{d o}^{*}, y_{r o}\right)$.

6.1. Theorem. The projection point for unit under assessment by model (6.1) is overall input-oriented efficient with convex intermediateproducts.

The proof of Theorem (6.1) appears in the Appendix. 
The dual of model (6.1) can be expressed as follows:

$$
\begin{array}{ll}
\max & \sum_{r=1}^{s} u_{r} y_{r o}+u_{0}+v_{0} \\
\text { s.t. } & \sum_{d=1}^{D} w_{d}^{1} z_{d j}-\sum_{i=1}^{m} v_{i} x_{i j}+v_{0} \leq 0 \quad j=1, \cdots, n \\
& \sum_{r=1}^{s} u_{r} y_{r j}-\sum_{d=1}^{D} w_{d}^{2} z_{d j}+u_{0} \leq 0 \quad j=1, \cdots, n \\
& \sum_{i=1}^{m} v_{i} x_{i o}=1 \\
& \sum_{d=1}^{D} w_{d}^{1}-\sum_{d=1}^{D} w_{d}^{2} \geq 0 \\
& v_{i} \geq 0, u_{r} \geq 0 \quad i=1, \cdots, m ; r=1, \cdots, s \\
& u_{0}, v_{0}, w^{2}, w^{1} \text { free in sign }
\end{array}
$$

The fractional program of model (6.2) can be expressed as:

$$
\begin{array}{ll}
\max & \frac{\sum_{r=1}^{s} u_{r} y_{r o}+u_{0}+v_{0}}{\sum_{i=1}^{m} v_{i} x_{i o}} \\
\text { s.t. } & \frac{\sum_{d=1}^{D} w_{d}^{1} z_{d j}+v_{0}}{\sum_{i=1}^{m} v_{i} x_{i j}} \leq 1 \quad j=1, \ldots, n \\
& \frac{\sum_{r=1}^{s} u_{r} y_{r j}+u_{0}}{\sum_{d=1}^{D} w_{d}^{2} z_{d j}} \leq 1 \quad j=1, \cdots, n \\
& \sum_{d=1}^{D} w_{d}^{1}-\sum_{d=1}^{D} w_{d}^{2} \geq 0 \\
& v_{i} \geq 0, u_{r} \geq 0 \quad i=1, \cdots, m ; r=1, \cdots, s \\
& u_{0}, v_{0}, w^{2}, w^{1} \text { free in sign }
\end{array}
$$

Here, $\tilde{z}_{d o}$ imposes the third set of constraints $\left(\sum_{d=1}^{D} w_{d}^{1}-\sum_{d=1}^{D} w_{d}^{2} \geq 0\right)$ to model (6.3). Note that these constraints are not redundant. This means that the cost of intermediate products considered as inputs is smaller or equal than to the cost when the same products were considered as outputs.

In fact, the problem is optimized in such a way that the price of providing intermediate products in the second stage equals, at most, to the price of selling the same product in the first stage.

\section{Illustrative examples}

In this section, the suggested models are used to assess overall and divisional efficiency scores and to determine frontier projections. In addition, we compare the findings of this study with some other two-stage network models.

First, we consider the data given in Table 1 and solve the models (6.1) and (2.5), which were proposed by Chen et al. [5], under the assumption of VRS. Then, using the results, we calculate the frontier projection units. The results are reported in Table 3.

The results show that the frontier projections determined by model (6.1) are exactly the same those obtained by model (2.5). In addition, when calculating the overall 
efficiency score of model (4.1) for $D M U_{B}$, due to applying the restriction on the intermediate products, this unit is efficient. However, with permission to change the convex hull of the intermediate products in model (6.1) the possibility of further abatement is created in inputs. Therefore, $D M U_{B}$ is inefficient under the model (6.1).

Table 3. Frontier projection results for four units in example 3.3

\begin{tabular}{|c|c|c|c|c|c|c|}
\hline \multirow[t]{2}{*}{ DMU } & \multicolumn{3}{|c|}{ MODEL (6.1) } & \multicolumn{3}{|c|}{ MODEL (2.5) } \\
\hline & $\theta^{*} \mathbf{x}$ & $\widetilde{\mathbf{z}}^{*}$ & $\mathrm{y}$ & $\theta^{*} \mathbf{x}$ & $\widetilde{\mathbf{z}}^{*}$ & $\mathrm{y}$ \\
\hline $\mathbf{A}$ & 1 & 2 & 4 & 1 & 2 & 4 \\
\hline $\mathbf{B}$ & 1 & 2 & 1 & 1 & 2 & 1 \\
\hline $\mathbf{C}$ & 1 & 2 & 2 & 1 & 2 & 2 \\
\hline D & 1 & 2 & 2 & 1 & 2 & 2 \\
\hline
\end{tabular}

To examine divisional efficiency scores in Example 3.3, we use Models (5.2) and (5.4). To compare results, we solve the proposed models by Kao and Hwang [13], under VRS assumption. The results are reported in Tables 4 and 5 .

As can be seen in Tables 4 and 5, the efficiency scores of first stage given by the model proposed in this study are greater than or equal to those obtained based on the model proposed by Kao and Hwang [13].

Table 4. Efficiency scores of four units in first stage for example 3.3

\begin{tabular}{|c|c|c|}
\hline DMU & MODEL (5.2) & MODEL (2.2) \\
\hline A & 1 & 1 \\
\hline B & 1 & 0.5 \\
\hline C & 1 & 1 \\
\hline D & 0.5 & 0.5 \\
\hline
\end{tabular}


Table 5. Efficiency scores of four units in second stage for Example 3.3

\begin{tabular}{|c|c|c|}
\hline DMU & MODEL (5.4) & MODEL (2.3) \\
\hline A & 1 & 1 \\
\hline B & 1 & 1 \\
\hline C & 0.33333 & 0.5 \\
\hline D & 0.666667 & 0.5 \\
\hline
\end{tabular}

7.1. Example. In this example, we evaluate the overall efficiency and divisional efficiency for a two-stage system using 12 DMUs. Two inputs, three intermediate products, and two outputs using hypothetical values are used in this evaluation. The data set is shown in Table 6.

Table 6. Data set of Example 7.1

\begin{tabular}{|c|c|c|c|c|c|c|c|}
\hline DMU & $\mathbf{x}_{\mathbf{1}}$ & $\mathbf{x}_{\mathbf{2}}$ & $\mathbf{z}_{\mathbf{1}}$ & $\mathbf{z}_{\mathbf{2}}$ & $\mathbf{z}_{\mathbf{3}}$ & $\mathbf{y}_{\mathbf{1}}$ & $\mathbf{y}_{\mathbf{2}}$ \\
\hline $\mathbf{1}$ & 61 & 5 & 3 & 12 & 4 & 10 & 75 \\
\hline $\mathbf{2}$ & 14 & 23 & 15 & 21 & 90 & 3 & 42 \\
\hline $\mathbf{3}$ & 25 & 10 & 6 & 50 & 12 & 6 & 6 \\
\hline $\mathbf{4}$ & 8 & 4 & 41 & 3 & 6 & 7 & 18 \\
\hline $\mathbf{5}$ & 53 & 20 & 18 & 14 & 55 & 90 & 10 \\
\hline $\mathbf{6}$ & 22 & 70 & 19 & 9 & 11 & 1 & 12 \\
\hline $\mathbf{7}$ & 17 & 55 & 40 & 20 & 15 & 30 & 14 \\
\hline $\mathbf{8}$ & 33 & 82 & 91 & 16 & 8 & 25 & 16 \\
\hline $\mathbf{9}$ & 25 & 45 & 6 & 10 & 78 & 7 & 38 \\
\hline $\mathbf{1 0}$ & 66 & 19 & 16 & 1 & 10 & 66 & 20 \\
\hline $\mathbf{1 1}$ & 21 & 64 & 9 & 8 & 15 & 22 & 42 \\
\hline $\mathbf{1 2}$ & 30 & 75 & 70 & 12 & 9 & 36 & 18 \\
\hline
\end{tabular}

The calculated overall and divisional efficiency scores of the units using models (4.1), (5.2), and (5.4) are reported in Table 7. The overall efficiency scores in most units are the same and are equal to one. Therefore, most units are efficient and lie on the VRS frontier, when they are evaluated by the new network model. These results are not surprising, as the production possibility set with the convex hull in intermediate products is limited. Significantly, the results shown in Table 7 revealed the equality of the overall efficiency scores with the efficiency scores for the first stage. 
Table 7. Overall and divisional efficiency scores results in Example 7.1

\begin{tabular}{|c|c|c|c|}
\hline DMU & Overall & stage 1 & stage 2 \\
\hline $\mathbf{1}$ & 1 & 1 & 1 \\
\hline $\mathbf{2}$ & 1 & 1 & 1 \\
\hline $\mathbf{3}$ & 1 & 1 & 1 \\
\hline $\mathbf{4}$ & 1 & 1 & 1 \\
\hline $\mathbf{5}$ & 0.7860 & 0.7860 & 1 \\
\hline $\mathbf{6}$ & 0.9077 & 0.9077 & 0.6491 \\
\hline $\mathbf{7}$ & 1 & 1 & 0.4292 \\
\hline $\mathbf{8}$ & 1 & 1 & 1 \\
\hline $\mathbf{9}$ & 1 & 1 & 1 \\
\hline $\mathbf{1 0}$ & 1 & 1 & 1 \\
\hline $\mathbf{1 1}$ & 1 & 1 & 0.9346 \\
\hline $\mathbf{1 2}$ & 0.7816 & 0.7816 & 0.9346 \\
\hline
\end{tabular}

By using the optimal solution for model (6.1) for $D M U_{o}$, the computed efficient projection of $\left(\theta^{*} x_{i o}, \tilde{z}_{d o}^{*}, y_{r o}\right)$ revealed the improved activity shown in Table 8 . Clearly, with the allowable change inthe convex hull of intermediate products, the possibility of further improvement is created in the units.

Table 8. Frontier projection results in Example 7.1

\begin{tabular}{|c|c|c|c|c|c|c|c|}
\hline $\mathbf{D M U}$ & $\theta^{*} \mathbf{x}_{\mathbf{1}}$ & $\theta^{*} \mathbf{x}_{\mathbf{2}}$ & $\widetilde{\mathbf{z}}_{\mathbf{1}}^{*}$ & $\widetilde{\mathbf{z}}_{\mathbf{2}}^{*}$ & $\widetilde{\mathbf{z}}_{\mathbf{3}}^{*}$ & $\mathbf{y}_{\mathbf{1}}$ & $\mathbf{y}_{\mathbf{2}}$ \\
\hline $\mathbf{1}$ & 61 & 5 & 3 & 12 & 4 & 10 & 75 \\
\hline $\mathbf{2}$ & 10.48 & 17.22 & 35.87 & 9.88 & 6.87 & 3 & 42 \\
\hline $\mathbf{3}$ & 10 & 4 & 41 & 3 & 6 & 6 & 6 \\
\hline $\mathbf{4}$ & 8 & 4 & 41 & 3 & 6 & 7 & 18 \\
\hline $\mathbf{5}$ & 41.65 & 15.72 & 18 & 14 & 55 & 90 & 10 \\
\hline $\mathbf{6}$ & 7.98 & 25.41 & 41 & 3 & 6 & 1 & 12 \\
\hline $\mathbf{7}$ & 8.48 & 27.44 & 39.73 & 4.22 & 7.67 & 30 & 14 \\
\hline $\mathbf{8}$ & 8.38 & 20.82 & 40.01 & 3.95 & 7.31 & 25 & 16 \\
\hline $\mathbf{9}$ & 10.07 & 18.13 & 36.72 & 8.73 & 6.73 & 7 & 38 \\
\hline $\mathbf{1 0}$ & 30.03 & 8.645 & 23.70 & 7.75 & 24.70 & 66 & 20 \\
\hline $\mathbf{1 1}$ & 10.62 & 32.38 & 35.56 & 10.30 & 6.93 & 22 & 42 \\
\hline $\mathbf{1 2}$ & 8.61 & 21.52 & 39.40 & 4.54 & 8.11 & 36 & 18 \\
\hline
\end{tabular}

\section{Conclusion}

Conventional DEA models with a two-stage network structure utilizing intermediate products, have different behaviors that this duality could impose limiting conditions on the pricing system. In this paper, we proposed uniform behavior using these intermediate products. For this purpose, we introduced a new overall production possibility set under the assumption of VRS, considering the convex hull of intermediate products. In addition, we proposed a network DEA model to assess the overall efficiency score and frontier projections. Then, we explained that the use of equality constraints in the intermediate product models decreased disposability, but due to considering separate and free variables in sign, or $w^{1}$ and $w^{2}$ within the dual models, the system allowed to price the intermediate products using different methods. Therefore, the proposed method is more flexible than 
conventional DEA models in a two-stage network structure. On the other hand, $\tilde{z}_{d o}$, in assessment of the model for frontier projections shows more compatibility with production assumptions. The main reason is that the model is optimized in such a way that the price of providing intermediate products in the second stage equals, at most, the price of selling the same product in the first stage. We examined the assessment methods for divisional efficiency in $T_{C H I}$, and explored that an assessment of divisional efficiency was not possible, because the efficiency of the first stage may represent the overall efficiency. Some illustrative examples were then applied to explain and compare the results of the approach presented here with those obtained by other methods.

\section{Appendix}

8.1. Theorem. The first stage technology or $T_{1}$, which is defined in set (3.1) is the minimal set that contains all observations and satisfies the axioms of strong disposability of inputs and convexity.

Proof. Assume technology $\mathrm{T}$ satisfies the axioms (A1)-(A3). We show that $T_{1} \subseteq T$. Namely, if activity $\left(x_{1}, z_{1}\right) \in T_{1}$ satisfies (A2) and (A3) with some vectors $\lambda \in R_{+}^{N}$ then $\left(x_{1}, z_{1}\right) \in T$. Let,

$$
\left(x_{1}, z_{1}\right) \in T_{1}, \exists \lambda_{1}, \cdots, \lambda_{n} \quad, \quad \sum_{j} \lambda_{j}=1:\left\{\begin{array}{l}
x_{1} \geq \sum_{j} \lambda_{j} x_{j} \\
z_{1}=\sum_{j} \lambda_{j} z_{j}
\end{array}\right.
$$

Since $\mathrm{T}$, satisfy (A1) then for any $\left(x_{j}, z_{j}\right) \in T, j=1, \cdots, N$. Also, T satisfies (A2) then we have :

$$
\sum_{j} \lambda_{j}\left(x_{j}, z_{j}\right) \in T \Rightarrow\left(\sum_{j} \lambda_{j} x_{j}, \sum_{j} \lambda_{j} z_{j}\right) \in T, \sum_{j} \lambda_{j}=1 .
$$

Finally, $\mathrm{T}$ must satisfy the strong disposability in inputs then, $\left(x_{1}, z_{1}\right) \in T$. Then, the proof is completed.

8.2. Theorem. The projection point for units under assessment given model (6.1) is overall input-efficient with intermediate convex products.

Proof. The efficiency of projection point obtained for $D M U_{o}$ based on model (6.1) namely $\left(\theta^{*} x_{i o}, \tilde{z}_{d o}^{*}, y_{r o}\right)$, is evaluated by solving model (4.1).

We have :

$\min \widehat{\theta}$

s. t. $\left(\widehat{\theta}\left(\theta^{*} x_{i o}\right), \tilde{z}_{o}^{*}, y_{o}\right) \in T_{C H I}$

We claim, $\widehat{\theta}^{*}=1$. Suppose not and let, $\widehat{\theta}^{*}<1$, (contrary hypothesis). Thus,

$$
\exists \widehat{\lambda} \geq 0,1 \widehat{\lambda}=1, \widehat{\mu} \geq 0,1 \widehat{\mu}=1 \rightarrow\left\{\begin{array}{l}
\sum_{j} \widehat{\lambda}_{j} x_{j} \leq \widehat{\theta}\left(\theta^{*} x_{o}\right) \\
\sum_{j} \widehat{\lambda}_{j} z_{j}=\tilde{z}_{o}^{*}=\sum_{j=1}^{N} \lambda_{j}^{*} z_{j} \\
\sum_{j} \widehat{\mu}_{j} z_{j}=\tilde{z}_{o}^{*}=\sum_{j=1}^{N} \mu_{j}^{*} z_{j} \\
\sum_{j} \widehat{\mu}_{j} y_{j} \geq y_{o}
\end{array}\right.
$$

Then $\left(\widehat{\theta} \theta^{*}, \widehat{\lambda}, \widehat{\mu}\right)$, is a feasible solution of model (4.1).

On the other hand according to contrary hypothesis we have $\widehat{\theta}^{*} \theta^{*}<\theta^{*}$. However, $\theta^{*}$ is part of an optimal solution and this is inconsistent with the optimality of $\theta^{*}$. Thus, $\widehat{\theta}^{*}=1$ and the proof is completed. 


\section{References}

[1] Charnes, A. Cooper, W.W. Rhodes, E. Measuring the efficiency of decision making units. European Journal of Operational Research 2, 429-444. 1978.

[2] Charnes, A. Cooper, W.W. Golany, B. Halek, R. Klopp, G. Schmitz, E. Thomas, D., Two phase data envelopment analysis approach to policy evaluation and management of army recruiting activities : Trade offs between Joint Services and Army Advertising. Research Report CCS no. 532, Center for Cybernetic Studies, The University of Texas at Austin, Texas. 1986.

[3] Chen, Y. Cook, W.D. Li, N. Zhu, J. Additive efficiency decomposition in two stage DEA. European Journal of Operational Research 96, 1170-1176. 2009a.

[4] Chen, Y. Liang, L. Zhu, J. Equivalence in two-stage DEA approaches. European Journal of Operational Research 193 (2), 600-604. 2009b.

[5] Chen, Y. Cook, W.D. Zhu, J. Deriving the DEA frontier for two-stage processes. European Journal of Operational Research 202, 138-142. 2010.

[6] Chen, Y. Cook, W. D. Kao, C. Zhu, J., Network DEA pitfall : Divisional efficiency and frontier projection under general network structures. European Journal of Operational Research $226,507-515.2013$.

[7] Cook, W. D. Seiford L. M. Data envelopment analysis (DEA) thirty years on. European Journal of Operational Research 192, 1-17. 2009.

[8] Ebrahimnejad, A., Tavana,M., Hosseinzadeh Lotfi, F., Shahverdi R. and Yousefpour,M., A three-stage data envelopment analysis model with application to banking industry, Mesurement, 49,308-319. 2014a.

[9] Ebrahimnejad E. and Tavana, M., An interactive MOLP method for identifying target units in Output-Oriented DEA models : The NATO enlargement problem, Measurement, 52, 124-134. 2014b.

[10] Ebrahimnejad, A., Bagherzadeh, M. R., Data envelopment analysis approach for discriminating efficient candidates in voting systems by considering the priority of voters, Hacettepe Journal of Mathematics and Statistics, 49 (1), 165-180. 2016.

[11] Hatami-Marbini, A. Ebrahimnejad, A. Lozano, S. Fuzzy efficiency measures in data envelopment analysis using lexicographic multiobjective approach, Computers \& Industrial Engineering, 105, 362-376. 2017.

[12] Kao, C. Hwang, S.-N. Efficiency decomposition in two-stage data envelopment analysis : an application to non-life insurance companies in Taiwan. European Journal of Operational Research185 (1), 418-429. 2008.

[13] Kao, C. Hwang, S.-N. Decomposition of technical and scale efficiencies in two-stage production systems. European Journal of Operational Research 211,515-519. 2011.

[14] Lovell, C.A.K. Walters, L.C. Wood, L.L. Stratified models of education production using modified DEA and regression analysis. In : Charnes, A. Liang, L. Cook, W.D. Zhu, J. 2008. DEA models for two-stage processes : game approach and efficiency decomposition. Naval Research Logistics 55, 643-653. 1994.

[15] Mottaghi, A. A. Ebrahimnejad, R. Ezzati, E. Khorram, A data envelopment analysis based approach for target setting and resource allocation : application in gas companies, Hacettepe Journal of Mathematics and Statistics, 45(5), 1553 -1578. 2016.

[16] Rho, s. An, J. Evaluating the efficiency of a two stage production process Using data envelopment analysis. International Transactions in Operational Research 14, 395-410. 2007.

[17] Seiford, L.M. Zhu, J. Profitability and marketability of the top 55 US commercial banks. Management Science 45, 1270-1288. 1999.

[18] Sexton, T.R. Lewis, H.F. Two-stage DEA : An application to major league baseball. Journal of Productivity Analysis 19, 227-249. 2003.

[19] Tavana, M., Ebrahimnejad, A., Santos-Arteaga, F. J., Mansourzadeh, S. M., Kazemi Matin, R. , A hybrid DEA-MOLP model for public school assessment and closure decision in the City of Philadelphia, Socio-Economic Planning Sciences, doi.org/10.1016/j.seps.2016.09.003. 2017.

[20] Tone, K. Tsutsui, M. Network DEA : a slacks-based measure approach. European Journal of Operational Research 197 (1), 243-252. 2009. 
[21] Wang, C. H. Gopal, R. Zionts. S. Use of data envelopment analysis in assess in information technology impact on firm performance. Annals of Operation Research73, 191-213. 1997. 